\title{
Interactions between "What" and "When" in the Auditory System: Temporal Predictability Enhances Repetition Suppression
}

\author{
Jordi Costa-Faidella, ${ }^{1,2}$ Torsten Baldeweg, ${ }^{3}$ Sabine Grimm, ${ }^{1,2}$ and Carles Escera ${ }^{1,2}$ \\ ${ }^{1}$ Institute for Brain, Cognition and Behavior (IR3C) and ${ }^{2}$ Cognitive Neuroscience Research Group, Department of Psychiatry and Clinical Psychobiology, \\ University of Barcelona, 08035 Barcelona, Catalonia, Spain, and ${ }^{3}$ Developmental Cognitive Neuroscience, Institute of Child Health, University College of \\ London, WC1N 1EH London, United Kingdom
}

Neural activity in the auditory system decreases with repeated stimulation, matching stimulus probability in multiple timescales. This phenomenon, known as stimulus-specific adaptation, is interpreted as a neural mechanism of regularity encoding aiding auditory object formation. However, despite the overwhelming literature covering recordings from single-cell to scalp auditory-evoked potential (AEP), stimulation timing has received little interest. Here we investigated whether timing predictability enhances the experience-dependent modulation of neural activity associated with stimulus probability encoding. We used human electrophysiological recordings in healthy participants who were exposed to passive listening of sound sequences. Pure tones of different frequencies were delivered in successive trains of a variable number of repetitions, enabling the study of sequential repetition effects in the AEP. In the predictable timing condition, tones were delivered with isochronous interstimulus intervals; in the unpredictable timing condition, interstimulus intervals varied randomly. Our results show that unpredictable stimulus timing abolishes the early part of the repetition positivity, an AEP indexing auditory sensory memory trace formation, while leaving the later part $(\sim>200 \mathrm{~ms})$ unaffected. This suggests that timing predictability aids the propagation of repetition effects upstream the auditory pathway, most likely from association auditory cortex (including the planum temporale) toward primary auditory cortex (Heschl's gyrus) and beyond, as judged by the timing of AEP latencies. This outcome calls for attention to stimulation timing in future experiments regarding sensory memory trace formation in AEP measures and stimulus probability encoding in animal models.

\section{Introduction}

The auditory system extracts stimulus probabilities from the acoustic scene to predict future events (Winkler et al., 2009): what do we expect on the basis of what we have heard before? Yet, guiding our actions in changing environments also involves anticipating when events will occur. Although the neural mechanisms of stimulus probability encoding have been the focus of much research, their interaction with stimulus timing is little understood.

Several studies showed that activity along sensory pathways is reduced with stimulus probability, a phenomenon supporting the neural representation of stimuli known as "repetition suppression" (Desimone, 1996). In the auditory system, repetition

\footnotetext{
Received May 25, 2011; revised 0ct. 17, 2011; accepted 0ct. 24, 2011

Author contributions: J.C.F., T.B., S.G., and C.E. designed research; J.C.F. and T.B. performed research; J.C.F., S.G., and C.E. contributed unpublished reagents/analytic tools; J.C.F. analyzed data; J.C.F., T.B., S.G., and C.E. wrote the paper.

This work was supported by Grant PSI2009-08063 and the Consolider Ingenio2010 Program (CDS2007-00012) from the Spanish Ministry of Science and Innovation, Grant 2009SGR11 from the Generalitat de Catalunya, and the ICREA Academia Distinguished Professorship awarded to C.E.

Correspondence should be addressed to Dr. Carles Escera, Cognitive Neuroscience Research Group, Department of Psychiatry and Clinical Psychobiology, University of Barcelona, P. Vall d'Hebron 171, 08035 Barcelona, Catalonia, Spain.E-mail: cescera@ub.edu.

DOI:10.1523/JNEUROSCI.2599-11.2011

Copyright $\odot 2011$ the authors $\quad 0270-6474 / 11 / 3118590-08 \$ 15.00 / 0$
}

suppression spans multiple spatial and time scales, as revealed by animal single-cell recordings exhibiting stimulus-specific adaptation (SSA) in cortical and subcortical structures (Ulanovsky et al., 2003, 2004; Pérez-González et al., 2005; Reches and Gutfreund, 2008; Anderson et al., 2009; Malmierca et al., 2009; Antunes et al., 2010; Farley et al., 2010; Zhao et al., 2011), human long- and middle-latency auditory-evoked potentials (AEP) (Haenschel et al., 2005; Slabu et al., 2010; Costa-Faidella et al., 2011; Grimm et al., 2011), and fMRI studies (Mutschler et al., 2010). However, none of the abovementioned studies explored the influence of timing regularity on repetition suppression, a subject lightly tapped in human electrophysiology literature, leading to contradictory findings. For example, whether single repeated tones in periodic versus aperiodic sequences elicit a smaller N1-P2 complex of the AEP is still unclear (Nelson et al., 1969; Rothman et al., 1970; Nelson and Lassman, 1977). Similarly, periodicity in an AEP oddball paradigm can yield N1 decrements (Harada et al., 2005), P50 decrements (Moberget et al., 2008), and contradictory effects on preattentive deviance detection (Takegata and Morotomi, 1999; Schwartze et al., 2011). Divergences might arise from using inappropriate stimulation paradigms; because AEP repetition effects occur rapidly, using different stimuli with a variable number of repetitions might prove more instructive (e.g., roving standard paradigm) (Baldeweg et al., 2004; Cowan et al., 1993). 


\section{Roving Standard Frequency Paradigm}

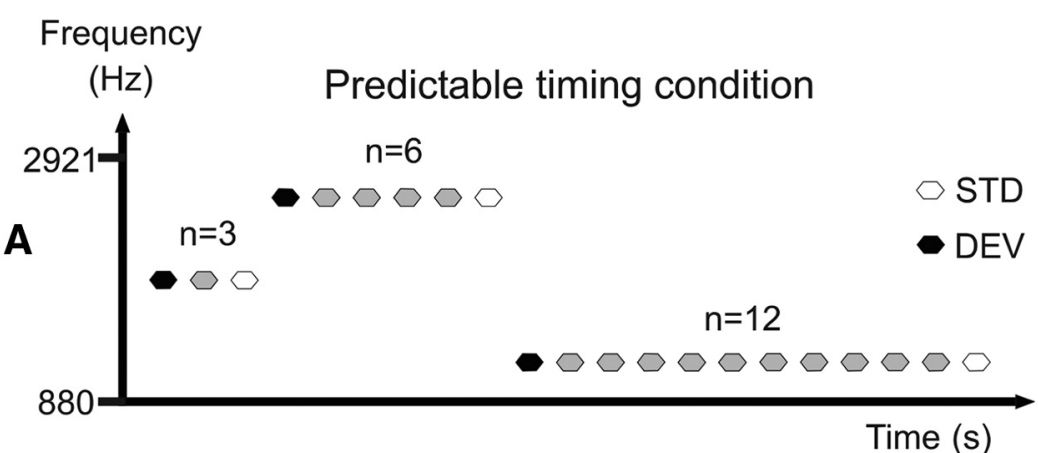

Unpredictable timing condition

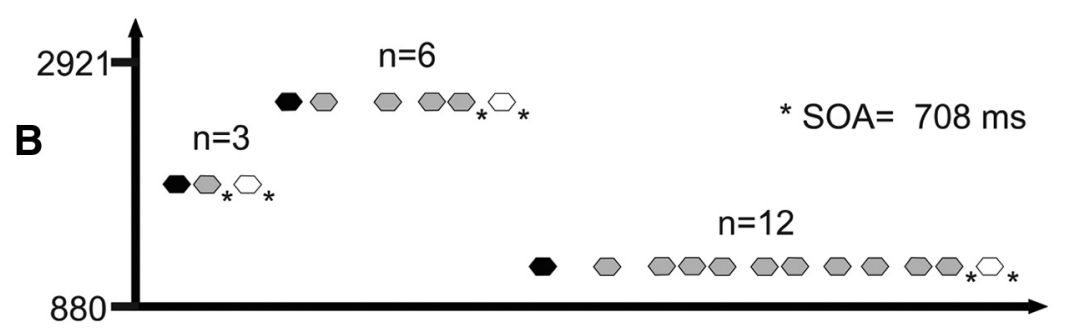

Figure 1. Schematic diagram of the roving standard frequency paradigm used in this study. Trains of three, six, or 12 equal tones were randomly delivered without intertrain pauses, with tone frequency varying from 880 to $2921 \mathrm{~Hz}$ across trains. In this arrangement, the first tone of a train acts as a low-probability stimulus compared with the previous train [deviant stimulus (DEV); black hexagons], whereas the last tone of a train acts as a high-probability stimulus inside that train [standard stimulus (STD); white hexagons]. A, Predictable timing condition. The SOA and the ITI were set constant at $708 \mathrm{~ms}$. $\boldsymbol{B}$, Unpredictable timing condition. The SOA varied pseudorandomly between 364 and $1062 \mathrm{~ms}$ in seven steps of $118 \mathrm{~ms}$, with the constraint that the SOA previous to the last stimulus in a train as well as the ITI were always 708 ms (asterisks).

Considering that recent perception theories contemplate repetition suppression as a neural correlate of the precision with which future sensory events can be predicted (i.e., suppression of prediction error) based on past stimulation history (Friston, 2005; Winkler et al., 2009), it is of high interest to assess the relevance of timing predictability in neural adaptation. The present study seeks to provide human electrophysiological evidence showing that temporal predictability enhances the experience-dependent modulation of neural activity associated with probabilistic stimuli.

Here we recorded human AEP to pure tones arranged in a passive roving standard paradigm delivered at isochronous or random time intervals. We aimed to obtain a combined modulation of AEPs generated along the auditory pathway (P50, N1, $\mathrm{P} 2$ ), conforming the repetition positivity (RP), an AEP-reflecting auditory sensory memory trace formation (Haenschel et al., 2005). If repetition suppression is enhanced by stimulus predictability, repetition effects should be greater in isochronous sound sequences. Furthermore, violating probability-based expectancies involving predictable time information should elicit stronger error signals, as indexed by the mismatch negativity (MMN) (Näätänen, 2007).

\section{Materials and Methods}

Participants. Seventeen healthy volunteers (6 male; age, 23-49 years; mean age, 29.18 years; all right-handed) with no history of neurological, psychiatric, or hearing impairment and with normal or corrected-tonormal visual acuity participated in the experiment. All volunteers gave informed consent before their participation. Data from two participants had to be excluded from the analysis; one due to a poor signal-to-noise ratio ( $<50 \%$ of artifact-free epochs in one block), and one due to a muscle artifact time-locked to the onset of the acoustic stimuli (picked up by the electrodes located at the mastoid positions). Ethical approval was obtained from the local ethical committee and all experiments were in accordance with the Declaration of Helsinki.

Stimuli and procedure. The auditory stimuli consisted of pure sinusoidal tones of $50 \mathrm{~ms} \mathrm{du}-$ ration, including $5 \mathrm{~ms}$ rise and fall times, generated with Audacity free software (version 1.3; http://audacity.sourceforge.net/). The tones were delivered binaurally through headphones by Presentation software (version 0.70; www.neurobs.com). Each subject adjusted the loudness of the tones to a comfortable level, which was maintained throughout the recording session (average loudness, $\sim 80 \mathrm{~dB}$ SPL). This experiment used a modified version of the roving standard frequency paradigm described by Baldeweg et al. (2004). Here, trains of three, six, or 12 equal tones were randomly delivered without intertrain pauses, with tone frequency varying across trains (Fig. 1). In such a stimulus arrangement, the first tone of a train acts as a low-probability stimulus compared with those of the previous train (deviant stimulus), whereas the last tone of a train acts as a highprobability stimulus inside that train (standard stimulus). This paradigm allowed us to derive two types of measures on the amount of change in the AEPs as a function of stimulus repetition: a direct measure of the adaptation to repetition, indexing sensory-memory trace formation (Haenschel et al., 2005) by comparing the AEPs to the last tone in a train of three, six, or 12 stimuli; and a measure of the neural activity related to deviance detection by means of the MMN, as obtained by subtracting the activity evoked by the standard stimulus from that evoked by the deviant stimulus (Näätänen et al., 1978). The reason for choosing trains of three rather than two stimulus presentations as used in previous studies with roving standard paradigms (Baldeweg et al., 2004, 2006; Haenschel et al., 2005) was to avoid the possibility of a residual MMN to either the preceding train of standards or to the deviant stimulus in the neural response to the repeated tone (Sams et al., 1983). Twenty-five different frequencies were used, ranging from 880 to $2921 \mathrm{~Hz}$, with a frequency ratio between adjacent tones of 0.05 according to the following formula: $\Delta f=(f 2-$ $f 1) /(f 2 \times f 1)^{1 / 2}$ (Ulanovsky et al., 2003). To avoid N1 refractoriness effects across trains, the tone frequency of a particular train did not appear in any of the 10 subsequent trains.

In the predictable timing condition, the stimulus onset asynchrony (SOA) and the intertrain interval (ITI) were set to $708 \mathrm{~ms}$. In the unpredictable timing condition, the SOA varied pseudorandomly between 364 and $1062 \mathrm{~ms}$ in seven steps of $118 \mathrm{~ms}$, equiprobably presented with the constraint that the SOA previous to the last stimulus in a train and the ITI were always $708 \mathrm{~ms}$ (Fig. $1 \mathrm{~B}$, asterisks). This constraint was adopted to avoid potential baseline confounds in the AEP analysis due to carryover effects from the AEP to the previous stimulus. In total, 150 trains of three, six, and 12 tone repetitions were delivered per condition (900 trains overall). Participants sat in a comfortable chair in a sound-attenuated and electrically shielded room. They were instructed to ignore the sounds and watch a silent movie with subtitles. The auditory stimuli were arranged in three blocks of predictable timing and three of unpredictable timing, delivered at random, with resting pauses in between. The total duration of the experiment was $\sim 80$ min, plus 1 h of EEG recording preparation.

Auditory-evoked potentials recording and analysis. The electroencephalogram (EEG) was continuously recorded with frequency limits of 0.05 $100 \mathrm{~Hz}$ and digitized at a sampling rate of $1000 \mathrm{~Hz}$ by a SynAmps amplifier (NeuroScan). Ag/AgCl electrodes were used for the EEG acquisition, 18 of which were mounted in a nylon cap (Quik-Cap; Compu- 
medics) at the standard locations Fp1, Fp2, F7, F3, Fz, F4, F8, T7, C3, Cz, C4, T8, P7, P3, Pz, P4, P8, and $\mathrm{Oz}$ according to the international 10-20 system. Additionally, two electrodes were positioned over the left and the right mastoids (M1 and M2, respectively). Vertical and horizontal electrooculogram (VEOG and HEOG, respectively) were measured from monopolar electrodes placed below (VEOG) and lateral (HEOG) to the right eye. The ground electrode was placed at Fpz and the common reference electrode was placed at Cpz. All impedances were kept $<5 \mathrm{k} \Omega$ during the whole recording session.

Data analysis was performed offline using EEGlab v.7 software (Delorme and Makeig, 2004) running under Matlab v7.6 (Mathworks). Continuous EEG data were resampled to $250 \mathrm{~Hz}$ and periods contaminated by nonstereotyped muscle artifacts were rejected by visual inspection. Independent component analysis decomposition was applied using the Infomax algorithm, removing blink-related independent components on the basis of their scalp topography and continuous activity (Jung et al., 2000). EOG artifact-corrected data were re-referenced to linked mastoids and filtered from 0.2 to $30 \mathrm{~Hz}$. Epochs of $600 \mathrm{~ms}$, starting $100 \mathrm{~ms}$ before stimulus onset and baseline corrected from -100 to $0 \mathrm{~ms}$, were extracted and averaged for each experimental condition separately ( 12 conditions; standard and deviant stimuli $\times$ predictable/unpredictable timing $\times 3$-, 6-, or 12-tone repetition trains; 150 epochs per condition). Before averaging, epochs exceeding an amplitude threshold of $\pm 100 \mu \mathrm{V}$ were rejected (mean of overall rejected epochs, 22; SD, 14.9). MMN difference waveforms were obtained by subtracting the activity elicited by the last stimulus in a train (i.e., standard stimulus: third, sixth, or 12th) from that elicited by the first stimulus of the subsequent train (i.e., deviant stimulus). For illustration purposes, RP difference waveforms were obtained by subtracting the activity elicited by the third standard stimulus from that elicited by the 12th standard stimulus. To examine the early onset of AEP repetition effects, we computed the mean amplitude in the 60-80 ms time window around the P50 peak at Fz electrode for all standard and deviant AEPs. N1 peak amplitudes were retrieved from all standard and deviant AEPs by detecting the minimum amplitude values in the $80-180$ $\mathrm{ms}$ time window at Fz. Similarly, P2 peak amplitudes were retrieved from all standard AEPs by detecting the maximum amplitude values in the $120-280$ ms time window at Fz. P2 values were not retrieved from deviant stimuli due to a possible overlap with the ongoing MMN and P300 AEP components taking place in the response to improbable stimuli (deviants). Finally, to compare the differential activity between standard and deviant stimuli around the MMN range, we retrieved the mean amplitudes for both stimulus types in a $30 \mathrm{~ms}$ time window centered at the individual MMN peak at the Fz electrode (detected as the minimum value in the difference waveforms in the $80-250 \mathrm{~ms}$ time window).

Statistical analysis. Timing predictability and repetition effects and their interactions were assessed by means of repeated-measures ANOVAs with the factors Stimulus (standard vs deviant), Timing predictability (predictable vs unpredictable), and Repetition $(3,6,12)$ for all P50, N1, and MMN (time range) measures specified above. Effects on P2 values were computed with repeated-measures ANOVAs with the factors Timing predictability (predictable vs unpredictable) and Repetition (3, 6, 12). Subsequent repeated-measures ANOVAs were performed to assess interaction effects. Linear trends are reported when applicable. The Greenhouse-Geisser correction was applied when the assumption of sphericity was violated. Significant ANOVA effects (alpha level $=0.05$ ) are reported with the partial $\eta^{2}$ effect size measure.

To ascertain that stimulus probability and timing predictability affected the studied AEP (P50, N1, P2) and that the expected modulations were not due to other neural processes summing up at the scalp with those components, we computed a topographical measure of global dissimilarity (DISS) (Murray et al., 2008). DISS is an index of configuration differences between two electric fields, independent of their strength. This test provides a statistical means of determining whether the brain networks activated by two conditions differ. DISS equals the square root of the mean of the squared differences between the potentials measured at each electrode (average re-referenced; note that the relative form of the scalp topography is reference-independent), each of which is first scaled to unitary strength by dividing by the instantaneous global field power (the root mean square across the average-referenced electrode values at a given instant in time). DISS can range from 0 (topographic homogeneity) to 2 (topographic inversion). Because DISS is a single measure of the distance between two vectors, a nonparametric statistical test has to be conducted and only pairwise comparisons are allowed with this method. We computed all possible pairwise comparisons between the scalp topographies obtained with the abovementioned measures for the P50, N1, and P2 AEPs, as follows: (1) data were average-referenced, (2) singlesubject maps were reassigned to different experimental conditions (i.e., permutations of the data), (3) grand-average AEPs were recalculated, (4) and a DISS value was recalculated for the new grand-average AEP. Five thousand permutations of the data were used to obtain the empirical distribution, and the alpha level to determine whether the observed DISS between two conditions was significantly different compared with the distribution was set to 0.05 .

\section{Results}

Grand average waveforms evoked to standard and deviant stimuli after three, six, and 12 stimulus presentations for both predictable and unpredictable timing conditions are illustrated in Figure $2 \mathrm{~A}$, together with deviant minus standard difference waveforms (Fz electrode). As expected, tones in both conditions elicited the typical AEP waveforms with distinct P50, N1, and P2 components, and the subtraction of the waveforms to low from high probability tones revealed a prominent MMN. Below, we describe in detail the influence of stimulus probability and timing predictability in these AEP components.

\section{Interactions between timing predictability and stimulus repetition effects on brain potentials}

Early effects at the $P 50$ range $(\sim 70 \mathrm{~ms})$

The P50 component of the AEP to standard and deviant stimuli indexed changes of early brain activity to tone repetition that depended on timing predictability (Timing predictability $\times$ Repetition interaction: $\left.F_{(2,28)}=3.536, p=0.043, \eta^{2}=0.202\right)$. In the predictable timing condition, the P50 mean amplitude evoked to standard and deviant stimuli was similar and increased as a function of repetition (Stimulus type: $F_{(1,14)}=0.640, p=0.437$; Stimulus type $\times$ Repetition interaction: $F_{(2,28)}=0.184, p=0.833$; Repetition effect for standard and deviant stimuli; $F_{(2,28)}=8.685$, $p=0.001 ; \eta^{2}=0.383$; linear trend: $F_{(1,14)}=22.601, p=0.0003$; $\eta^{2}=0.617$ ), but it showed no significant changes in the unpredictable timing condition (Stimulus type: $F_{(1,14)}=0.954, p=$ 0.345 ; Stimulus type $\times$ Repetition interaction: $F_{(2,28)}=1.901$, $p=0.168$; Repetition effect for standard and deviant stimuli: $\left.F_{(2,28)}=0.066, p=0.936\right)$. The topographical distribution of the P50 AEP remained stable across stimulus types and repetitions and did not depend on timing predictability, according to DISS (Murray et al., 2008) on every possible pairwise comparison. Stimulus repetition-related changes in P50 mean amplitude elicited to deviant and standard stimuli in both predictable and unpredictable conditions are illustrated in Figure $2 B$.

Effects at the $\mathrm{N} 1$ range $(\sim 110 \mathrm{~ms})$

When analyzing the peak amplitudes of the N1 component of the AEP, larger amplitudes were found for deviant than standard stimuli (Stimulus type main effect: $F_{(1,14)}=18.308, p=0.001$, $\left.\eta^{2}=0.567\right)$. This main effect interacted with repetition (Stimulus type $\times$ Repetition interaction: $F_{(2,28)}=5.066, p=0.009, \eta^{2}=$ $0.285)$. Thus, when analyzing the N1 peak amplitude separately for deviant stimuli, no significant effects were found (Timing predictability: $F_{(1,14)}=0.916, p=0.355$; Repetition: $F_{(2,28)}=$ $0.940, p=0.403$; Timing predictability $\times$ Repetition interaction: $\left.F_{(2,28)}=0.027, p=0.974\right)$, but the repetition effect interacted with timing predictability for standard stimuli (Timing predict- 
A
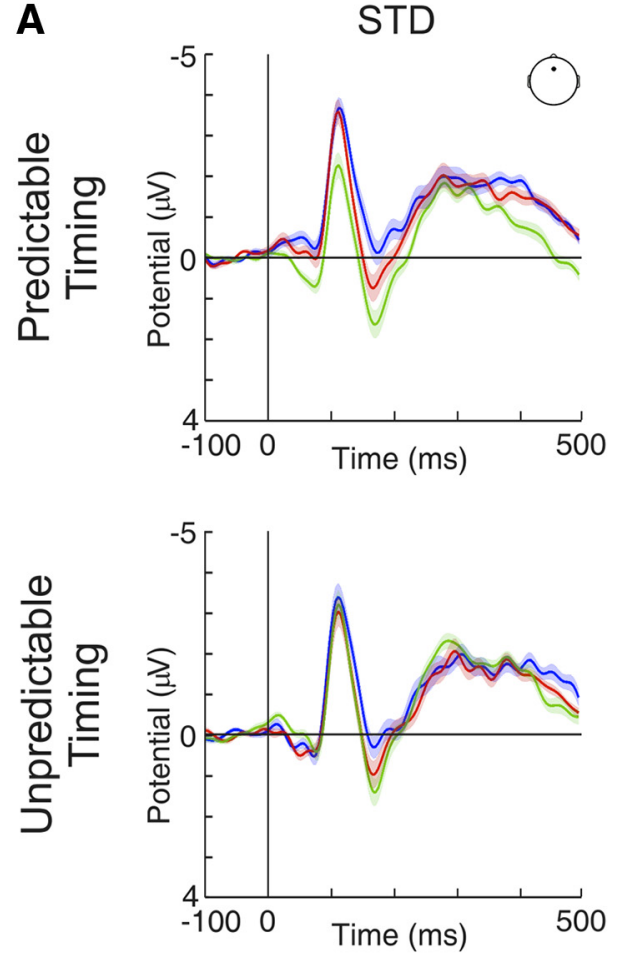

DEV
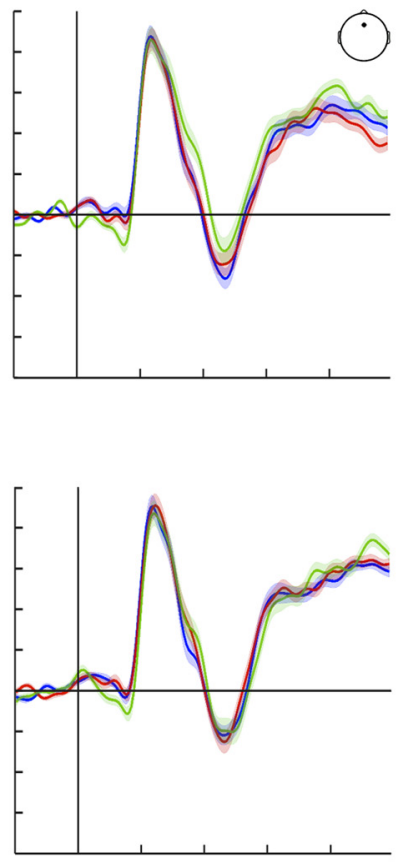

DEV-STD (DW)
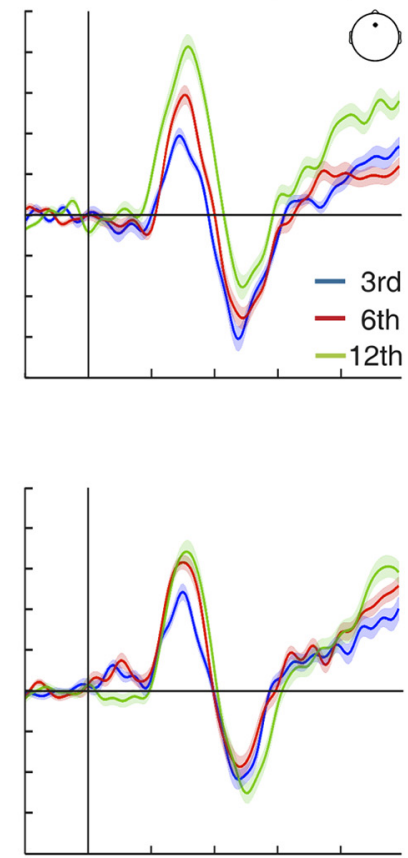

B

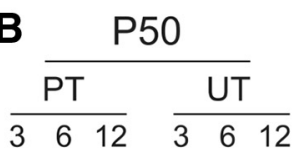

C

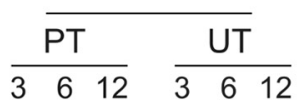

D
E MMNwin

$\frac{\mathrm{PT}}{3612} \frac{\text { UT }}{3612}$
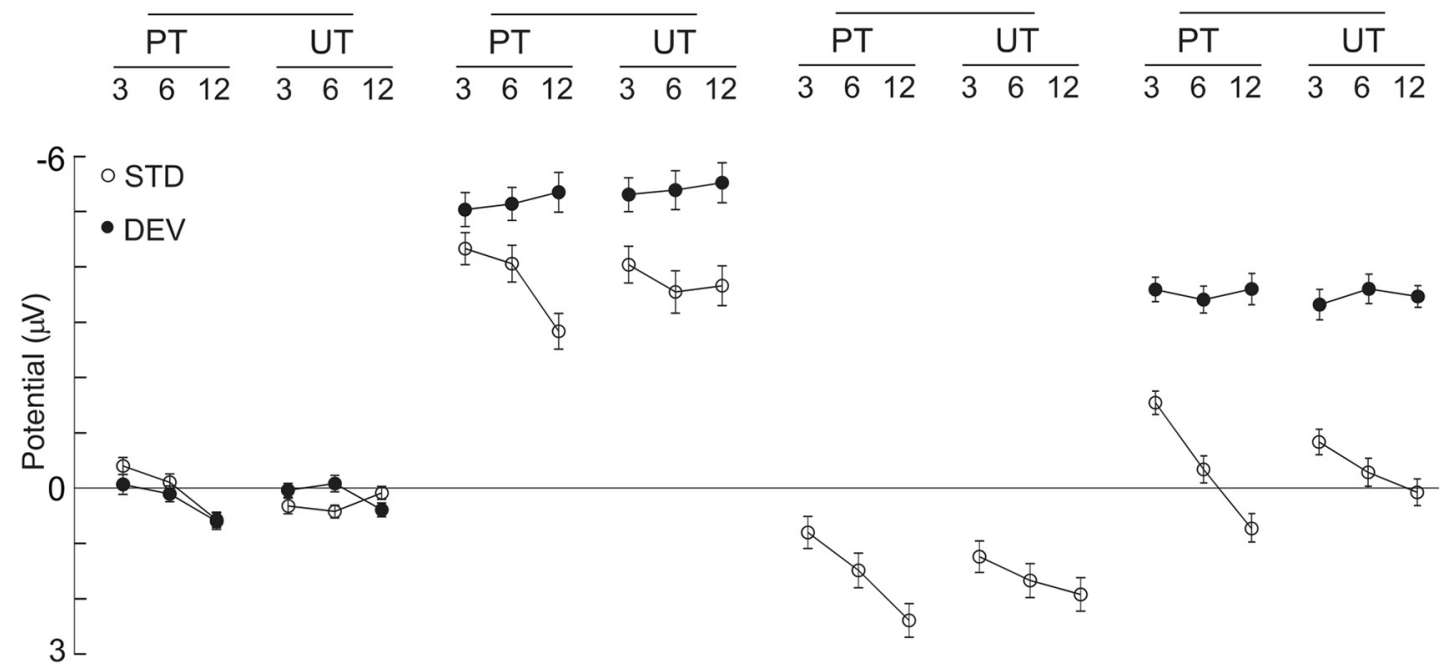

Figure 2. A, Grand-average waveforms for standard (STD), deviant (DEV), and deviant minus standard differences (DEV-STD DW) in predictable (top) and unpredictable (bottom) timing conditions, separately for trains of three (blue trace), six (red trace), and 12 (green trace) tone presentations, as recorded from Fzelectrode. Shaded areas indicate SEM. B, P50 amplitudes in predictable (PT) and unpredictable (UT) timing conditions elicited to standard (white circles) and deviant (black circles) stimuli separately for trains of three, six, and 12 tones (amplitudes in microvolts; error bars denote SEM). P50 amplitude increased with repetition only in the predictable timing condition regardless of stimulus type. $\boldsymbol{C}$, Same as $\boldsymbol{B}$, but for $\mathrm{N} 1$ amplitudes, which were overall larger for deviant than standard stimuli but decreased with further repetition only for standard stimuli in the predictable timing condition. $\boldsymbol{D}$, Same as $\boldsymbol{B}$, but for P2 amplitudes elicited to the standard stimulus. P2 amplitudes increased with tone repetition regardless of timing predictability. $\boldsymbol{E}$, Same as $\boldsymbol{B}$, but for amplitudes retrieved in a time window around the MMN. Deviant stimuli elicited more negative amplitudes in the MMN time window (MMNwin) than standard stimuli, but only the latter were affected by repetition, an effect manifested as an increase of positivity, larger in the predictable than the unpredictable timing condition.

ability $\times$ Repetition interaction: $F_{(2,28)}=4.786, p=0.016$, $\left.\eta^{2}=0.255\right)$. This repetition effect was present in the predictable timing condition (Repetition main effect: $F_{(2,28)}=11.123, \varepsilon=$ $0.706, p=0.001, \eta^{2}=0.443$; linear trend: $F_{(1,14)}=14.633, p=$ $\left.0.002, \eta^{2}=0.511\right)$ but not in the unpredictable timing condition $\left(F_{(2,28)}=0.846, p=0.440\right)$. The topographical distribution of the N1 AEP remained stable across stimulus types and repetitions and did not depend on timing predictability according to a DISS analysis on every possible pairwise comparison. N1 peak amplitudes retrieved from standard and deviant stimuli in both predictable and unpredictable timing conditions are plotted as a function of stimulus repetition in Figure 2C.

Effects at the $P 2$ range $(\sim 170 \mathrm{~ms})$

The analysis of the peak amplitude of the P2 component of the auditory AEP to standard stimuli yielded an increase with repe- 
tition regardless of timing predictability, as shown by a significant effect of stimulus repetition without an interaction with timing predictability (Interaction: $F_{(2,28)}$ $=2.334, p=0.116$; Repetition main effect: $F_{(2,28)}=4.899, \varepsilon=0.706, p=0.028$, $\eta^{2}=0.259$; linear trend: $F_{(1,14)}=6.108$, $\left.p=0.027, \eta^{2}=0.304\right)$. The topographical distribution of the P2 AEP remained stable across stimulus repetitions and did not depend on timing predictability according to a DISS analysis on every possible pairwise comparison. These changes in $\mathrm{P} 2$ peak amplitude with stimulus repetition in both predictable and unpredictable timing conditions are illustrated in Figure $2 D$.

In addition to the above mentioned analyses, the effect of stimulus repetition on the standard stimuli AEPs and its interaction with stimulus timing can be better seen in Figure $3 A$ where, for illustration purposes, we subtracted the activity elicited by the third from that elicited by the 12 th tone in a train, a procedure commonly used to obtain the RP (Haenschel et al., 2005). The figure shows that the RP develops with stimulus repetition at much earlier latencies in the predictable timing (Fig. $3 A$, blue trace) than in the unpredictable timing (Fig. $3 A$, red trace) condition, an effect highlighted by the color arrows in the figure, marking the time windows of the P50 $(\sim 70 \mathrm{~ms}$; Fig. $3 A$, blue arrow $)$ and $\mathrm{P} 2(\sim 170 \mathrm{~ms}$; Fig. $3 A$, red arrow) components found in the standard stimuli AEPs. The typically frontocentral scalp potential distribution of the RP (Haenschel et al., 2005) is plotted in Figure $3 B$ at the abovementioned time windows. This figure shows the lack of repetition effects in the AEP to standard stimuli over the whole scalp, until the $\mathrm{P} 2$ time range, in the unpredictable timing condition.

\section{Interactions between timing predictability and stimulus repetition effects in auditory deviance detection}

Auditory deviance detection was influenced both by stimulus repetition and timing predictability, as shown by an analysis of the separate contributions of deviant and standard stimuli to the MMN AEP (Fig. 2E). Repetition effects were modulated by stimulus type and timing predictability (triple interaction between Stimulus type $\times$ Timing predictability $\times$ Repetition: $F_{(2,28)}=$ $3.396, p=0.048, \eta^{2}=0.195$ ), being present mainly for standard stimuli and larger in the predictable than in the unpredictable timing conditions. Subsequent analyses showed that in the predictable timing condition, as expected, deviant stimuli elicited more negative amplitudes in the MMN time window overall than standard stimuli (Stimulus type main effect: $F_{(1,14)}=83.758, p=2.8 \times$ $\left.10^{-7}, \eta^{2}=0.857\right)$, although repetition effects were only present in the responses to the latter (Stimulus type $\times$ Repetition interaction: $F_{(2,28)}=10.875, p=0.0003, \eta^{2}=0.437$; Repetition effects on deviant stimuli: $F_{(2,28)}=0.159, p=0.853$; Repetition effects on standard stimuli: $F_{(2,28)}=9.109, p=0.001, \eta^{2}=0.394$; linear trend: $F_{(1,14)}=$ 15.093, $\left.p=0.002, \eta^{2}=0.519\right)$. In the unpredictable timing

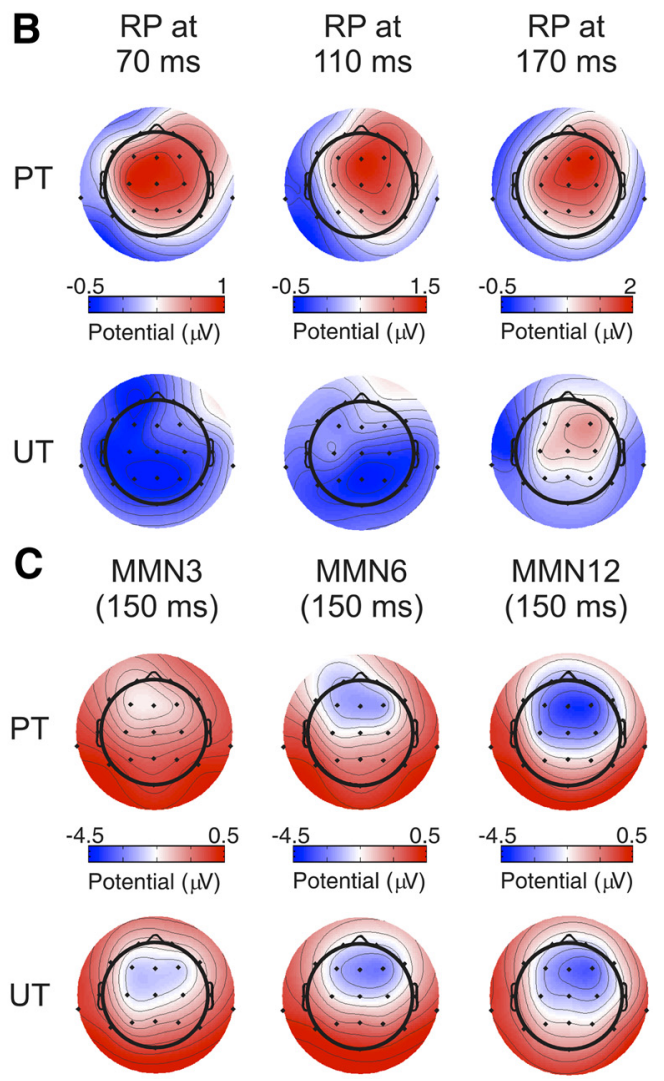

Figure 3. $\quad \boldsymbol{A}, \mathrm{RP}$ grand-average difference waveforms [AEP to the 12th minus the third standard (STD) stimulus] for predictable potential distribution after three, six, and 12 tone presentations in predictable and unpredictable timing conditions. Note the repetition-related increase in amplitude and the typical frontocentral distribution of the MMN (using linked mastoids reference).

condition, deviant stimuli elicited more negative amplitudes in the MMN time window overall than standard stimuli (Stimulus type main effect: $\left.F_{(1,14)}=79.480, p=3.8 \times 10^{-7}, \eta^{2}=0.850\right)$ and an interaction between Stimulus type and Repetition was also present $\left(F_{(2,28)}=3.977, p=0.030, \eta^{2}=0.221\right)$, indicating that repetition effects had opposite directions for deviant (increasing negativity) than standard (increasing positivity) stimuli. However, repetition effects, per se, did not reach significance in standard nor deviant stimuli (Repetition effects on deviant stimuli: $F_{(2,28)}=0.244, p=$ 0.785; Repetition effects on standard stimuli: $F_{(2,28)}=1.963, p=$ $0.159)$. Additionally, the typically frontocentral scalp potential distribution of the MMN (Alho, 1995; Näätänen and Alho, 1995) can be seen in Figure $3 C$ as a function of stimulus probability and timing predictability.

\section{Discussion}

The present study constitutes the first demonstration that timing predictability enhances the experience-dependent modulation of neural activity associated with stimulus probability encoding. Specifically, we have shown that isochronous stimulus repetition enhances the early part of the repetition positivity $(\sim<200 \mathrm{~ms})$, an AEP indexing auditory sensory-memory trace formation. This suggests that predictable timing aids the propagation of repetition effects upstream the auditory pathway, as judged by the timing of AEP latencies. Furthermore, violating probability-based 
stimulus expectancies involving regular time relations elicited a greater error signal, as reflected by larger MMN amplitudes in the predictable compared with the unpredictable timing condition.

Using roving standard stimulation, enabling the observation of repetition-related effects, Baldeweg and colleagues showed in several studies that tone repetition modulates the AEP components in a conjoined and reliable way: an increase of the P50, decrease of the N1, and increase of the P2 AEPs, which they called the repetition positivity (Baldeweg et al., 1999, 2006; Haenschel et al., 2005). Our data argue for the view of the RP as a nonunitary phenomenon, as the modulation of the underlying AEPs, supported by their stable scalp topographies across the different experimental conditions, might reflect different processing stages of regularity encoding in the auditory pathway.

Particularly, we showed that P2 amplitude increased with repetition regardless of the timing predictability of the sound sequence. P2 amplitude increases with stimulus repetition in timescales of minutes (Baldeweg et al., 1999) and days (Atienza et al., 2002), and correlates with stimulus expectancy driven by local and global stimulus probabilities (for a review of the P2 AEP, see Crowley and Colrain, 2004; Costa-Faidella et al., 2011). This suggests that P2, with neural generators localized to planum temporale (PT) as well as in area 22 (auditory association cortex) (Godey et al., 2001), might reflect the encoding of the "what" aspect of auditory stimulation, in line with the idea that the PT is a crucial structure in the generation of auditory objects (Griffiths and Warren, 2002).

However, the N1 behaved differently, increasing to tone changes regardless of timing predictability, but showing decrements with an increased number of stimulus repetitions only in isochronous sequences. Thus, the $\mathrm{N} 1$ evoked by a repeated tone is affected by the "when" aspect of auditory stimulation. Our data seem at odds with a study by Budd and colleagues (1998) in which trains of isochronous tones, including tone repetitions at the first five positions of each train, were delivered to healthy participants. The authors found that after the second repeated tone, N1 amplitude does not reveal a further decrease with repetition. Yet differences might arise from the use of a higher number of repeated stimuli in our paradigm, leading to a stronger memorytrace effect. The reason why this further N1 amplitude decrement only took place in our predictable timing condition needs more consideration. For example, N1 amplitude decreases with temporal and pitch expectations (Lange, 2009), with previous knowledge of the sequence of stimulation (Clementz et al., 2002), and to self-generated tones (Baess et al., 2011). The common aspect in these different studies is that they support the involvement of predictive mechanisms in $\mathrm{N} 1$ amplitude attenuation. Following this reasoning, our results show that increasing the predictability of the auditory stimulation both in stimulus probability and stimulus timing leads to a pronounced N1 attenuation (but see Nelson et al., 1969, 1977; Rothman et al., 1970; Harada et al., 2005).

As with the N1, repetition effects of the P50 were only observed in isochronous sequences. The increase of P50 amplitude extends findings from previous studies using roving standard paradigms (Haenschel et al., 2005; Baldeweg et al., 2006) or embedding stimulus repetitions in changing acoustic backgrounds (Dyson et al., 2005). Here we showed that temporal regularity is a necessary requirement to elicit P50 repetition-related amplitude increments, strongly suggesting the existence of an inference generation mechanism involving the encoding of precise temporal contingencies (Clementz et al., 2002; Bendixen et al., 2009).

The fact that the P50 amplitude evoked by a deviant tone was not different from that evoked by its preceding standard supports the view that early AEPs reflect the attempt of the auditory system to predict the sound input in the immediate future (Bendixen et al., 2009), and that deviations from the predicted input are detected at later stages of stimulus processing, possibly reflected by the $\mathrm{N} 1(\sim 110 \mathrm{~ms})$ and the MMN $(\sim 150 \mathrm{~ms})$ (Näätänen and Winkler, 1999). Although the correlates of auditory deviance detection found in our study appear at these relatively long latencies (at $\sim 110 \mathrm{~ms}$ after deviance onset and onwards), recent studies have shown mismatch responses at the middle-latency AEPs ( $\sim 40 \mathrm{~ms}$ ) (Slabu et al., 2010; Grimm et al., 2011), supportive of a multistage deviance detection system in the auditory modality (Grimm and Escera, 2011). The disagreement with our results might arise from using shorter interstimulus intervals $(<300 \mathrm{~ms}$ compared with $708 \mathrm{~ms}$ used here), leading to stronger memorytrace effects on deviance detection.

The onset of RP in the latency of P50 ( $\sim 70 \mathrm{~ms}$; isochronous condition) implicates the primary auditory cortex in its generation, based on a latency comparison with intracranial generators of human AEP (Liegeois-Chauvel et al., 1991). This, with all caution in comparing different neural scales, makes the RP a possible human electrophysiological counterpart of SSA, with which it shares many properties: both occur without overt attention to sounds, are stimulus-specific, and develop rapidly over multiple timescales (Baldeweg, 2007; Nelken and Ulanovsky, 2007; CostaFaidella et al., 2011). Although SSA literature is overwhelming (Ulanovsky et al., 2003, 2004; Pérez-González et al., 2005; Reches and Gutfreund, 2008; Anderson et al., 2009; Malmierca et al., 2009; Antunes et al., 2010; Farley et al., 2010; Zhao et al., 2011), to date, no study has attempted to explore SSA-timing interactions. To confirm that an irregular timing dampens the repetition effects on a neuronal scale, further research in animal models tapping the influence of timing predictability in the generation of SSA should prove instructive.

In summary, our study shows that the more regular, and thus predictable, the pattern of incoming sounds is, the shorter the latency of the AEP components exhibiting repetition suppression. Because human AEPs show a systematic hierarchy with latencies up to $70 \mathrm{~ms}$ generated along Heschl's gyrus, and later peaks generated in belt and parabelt (planum temporale) areas (Godey et al., 2001), our data suggest that the degree of predictability aids the propagation of repetition suppression upstream the auditory pathway. This idea is not new: Baldeweg (2006) raised it under the term "back-propagation hypothesis," stating that, with increasing number of repetitions, a stimulus-specific memory trace could be detected at earlier auditory processing stages in a top-down fashion in line with a predictive coding account (Friston, 2005). We extend this notion by including timing as an important variable in the formation of stimulus-specific memory traces at the level of the primary auditory cortex and perhaps further upstream.

It is important to note that sensory-memory trace formation is dependent on short-term synaptic plasticity, mainly mediated via NMDA receptor function, which is also essential in the generation of MMN/RP (Javitt et al., 1996; Umbricht et al., 2000; Näätänen et al., 2011). The lack of RP associated with timing uncertainty in our study might thus suggest the existence of a beat-based mechanism promoting a fine temporal adjustment of active top-down predictive signals (Nobre et al., 2007). A plausible candidate for such a mechanism is the entrainment of brain oscillations to stimulus presentation rate (Lakatos et al., 2008). Rhythmical deflections in the membrane potential could shift the excitability (i.e., depolarization) in local neuronal ensembles (Lakatos et al., 2005), aiding stimulus processing and memory 
trace formation via NMDA receptor activation. Future research using time-frequency decompositions of the electroencephalographic data may shed light on the interplay between entrained neural oscillations to rhythmic stimulation and repetition suppression.

\section{References}

Alho K (1995) Cerebral generators of mismatch negativity (MMN) and its magnetic counterpart (MMNm) elicited by sound changes. Ear Hear $16: 38-51$

Anderson LA, Christianson GB, Linden JF (2009) Stimulus-specific adaptation occurs in the auditory thalamus. J Neurosci 29:7359-7363.

Antunes FM, Nelken I, Covey E, Malmierca MS (2010) Stimulus-specific adaptation in the auditory thalamus of the anesthetized rat. PloS One 5:e14071.

Atienza M, Cantero JL, Dominguez-Marin E (2002) The time course of neural changes underlying auditory perceptual learning. Learn Mem 9:138-150.

Baess P, Horváth J, Jacobsen T, Schröger E (2011) Selective suppression of self-initiated sounds in an auditory stream: an ERP study. Psychophysiology 48:1276-1283.

Baldeweg T (2006) Repetition effects to sounds: evidence for predictive coding in the auditory system. Trends Cogn Sci 10:93-94.

Baldeweg T (2007) ERP repetition effects and mismatch negativity generation: A predictive coding perspective. J Psychophysiol 21:204-213.

Baldeweg T, Williams JD, Gruzelier JH (1999) Differential changes in frontal and sub-temporal components of mismatch negativity. Int J Psychophysiol 33:143-148.

Baldeweg T, Klugman A, Gruzelier J, Hirsch SR (2004) Mismatch negativity potentials and cognitive impairment in schizophrenia. Schizophr Res 69:203-217.

Baldeweg T, Wong D, Stephan KE (2006) Nicotinic modulation of human auditory sensory memory: evidence from mismatch negativity potentials. Int J Psychophysiol 59:49-58.

Bendixen A, Schröger E, Winkler I (2009) I heard that coming: event-related potential evidence for stimulus-driven prediction in the auditory system. J Neurosci 29:8447-8451.

Budd TW, Barry RJ, Gordon E, Rennie C, Michie PT (1998) Decrement of the N1 auditory event-related potential with stimulus repetition: habituation vs. refractoriness. Int J Psychophysiol 31:51-68.

Clementz BA, Barber SK, Dzau JR (2002) Knowledge of stimulus repetition affects the magnitude and spatial distribution of low-frequency eventrelated brain potentials. Audiol NeuroOtol 7:303-314.

Costa-Faidella J, Grimm S, Slabu L, Díaz-Santaella F, Escera C (2011) Multiple time scales of adaptation in the auditory system as revealed by human evoked potentials. Psychophysiology 48:774-783.

Cowan N, Winkler I, Teder W, Näätänen R (1993) Memory prerequisites of mismatch negativity in the auditory event-related potential (ERP). J Exp Psychol Learn Mem Cogn 19:909-921.

Crowley KE, Colrain IM (2004) A review of the evidence for P2 being an independent component process: age, sleep and modality. Clin Neurophysiol 115:732-744.

Delorme A, Makeig S (2004) EEGLAB: an open source toolbox for analysis of single-trial EEG dynamics including independent component analysis. J Neurosci Methods 134:9-21.

Desimone R (1996) Neural mechanisms for visual memory and their role in attention. Proc Natl Acad Sci U S A 93:13494-13499.

Dyson BJ, Alain C, He Y (2005) I've heard it all before: perceptual invariance represented by early cortical auditory-evoked responses. Brain Res Cogn Brain Res 23:457-460.

Farley BJ, Quirk MC, Doherty JJ, Christian EP (2010) Stimulus-specific adaptation in auditory cortex is an NMDA-independent process distinct from the sensory novelty encoded by the mismatch negativity. J Neurosci $30: 16475-16484$.

Friston K (2005) A theory of cortical responses. Philos Trans R Soc Lond B Biol Sci 360:815-836.

Godey B, Schwartz D, de Graaf JB, Chauvel P, Liégeois-Chauvel C (2001) Neuromagnetic source localization of auditory evoked fields and intracerebral evoked potentials: a comparison of data in the same patients. Clin Neurophysiol 112:1850-1859.
Griffiths TD, Warren JD (2002) The planum temporale as a computational hub. Trends Neurosci 25:348-353.

Grimm S, Escera C (2011) Auditory deviance detection revisited: evidence for a hierarchical novelty system. Int J Psychophysiology. doi:10.1016/j.ijpsycho.2011.05.012.

Grimm S, Escera C, Slabu L, Costa-Faidella J (2011) Electrophysiological evidence for the hierarchical organization of auditory change detection in the human brain. Psychophysiology 48:377-384.

Haenschel C, Vernon DJ, Dwivedi P, Gruzelier JH, Baldeweg T (2005) Event-related brain potential correlates of human auditory sensory memory-trace formation. J Neurosci 25:10494-10501.

Harada N, Masuda T, Endo H, Nakamura Y, Takeda T, Tonoike M (2005) The effect of $1 /$ f fluctuation in inter-stimulus intervals on auditory evoked mismatch field. Neurosci Lett 379:223-228.

Javitt DC, Steinschneider M, Schroeder CE, Arezzo JC (1996) Role of cortical N-methyl-D-aspartate receptors in auditory sensory memory and mismatch negativity generation: implications for schizophrenia. Proc Natl Acad Sci U S A 93:11962-11967.

Jung TP, Makeig S, Westerfield M, Townsend J, Courchesne E, Sejnowski TJ (2000) Removal of eye activity artifacts from visual event-related potentials in normal and clinical subjects. Clin Neurophysiol 111:1745-1758.

Lakatos P, Shah AS, Knuth KH, Ulbert I, Karmos G, Schroeder CE (2005) An oscillatory hierarchy controlling neuronal excitability and stimulus processing in the auditory cortex. J Neurophysiol 94:1904-1911.

Lakatos P, Karmos G, Mehta AD, Ulbert I, Schroeder CE (2008) Entrainment of neuronal oscillations as a mechanism of attentional selection. Science 320:110-113.

Lange K (2009) Brain correlates of early auditory processing are attenuated by expectations for time and pitch. Brain Cogn 69:127-137.

Liegeois-Chauvel C, Musolino A, Chauvel P (1991) Localization of the primary auditory area in man. Brain 114:139-151.

Malmierca MS, Cristaudo S, Pérez-González D, Covey E (2009) Stimulusspecific adaptation in the inferior colliculus of the anesthetized rat. J Neurosci 29:5483-5493.

Moberget T, Karns CM, Deouell LY, Lindgren M, Knight RT, Ivry RB (2008) Detecting violations of sensory expectancies following cerebellar degeneration: a mismatch negativity study. Neuropsychologia 46:2569-2579.

Murray MM, Brunet D, Michel CM (2008) Topographic ERP analyses: a step-by-step tutorial review. Brain Topogr 20:249-264.

Mutschler I, Wieckhorst B, Speck O, Schulze-Bonhage A, Hennig J, Seifritz E, Ball T (2010) Time scales of auditory habituation in the amygdala and cerebral cortex. Cereb Cortex 20:2531-2539.

Näätänen R (2007) The mismatch negativity: where is the big fish? J Psychophysiol 21:133-137.

Näätänen R, Alho K (1995) Generators of electrical and magnetic mismatch responses in humans. Brain Topogr 7:315-320.

Näätänen R, Winkler I (1999) The concept of auditory stimulus representation in cognitive neuroscience. Psychol Bull 125:826-859.

Näätänen R, Gaillard AW, Mäntysalo S (1978) Early selective-attention effect on evoked potential reinterpreted. Acta Psychologica 42:313-329.

Näätänen R, Kujala T, Kreegippu K, Carlson S, Escera C, Baldeweg T, Ponton C (2011) The mismatch negativity: an index of cognitive decline in neuropsychiatric and neurological diseases and in aging. Brain. doi:10.1093/brain/awr064.

Nelken I, Ulanovsky N (2007) Mismatch negativity and stimulus-specific adaptation in animal models. J Psychophysiol 21:214-223.

Nelson DA, Lassman FM (1977) Re-examination of the effects of periodic and aperiodic stimulation on the auditory-evoked vertex response. Audiology 16:409-418.

Nelson DA, Lassman FM, Hoel RL (1969) The effects of variable-interval and fixed-interval signal presentation schedules on the auditory evoked response. J Speech Hear Res 12:199-209.

Nobre A, Correa A, Coull J (2007) The hazards of time. Curr Opin Neurobiol 17:465-470.

Pérez-González D, Malmierca MS, Covey E (2005) Novelty detector neurons in the mammalian auditory midbrain. Eur J Neurosci 22:2879-2885.

Reches A, Gutfreund Y (2008) Stimulus-specific adaptations in the gaze control system of the barn owl. J Neurosci 28:1523-1533.

Rothman HH, Davis H, Hay IS (1970) Slow evoked cortical potentials and temporal features of stimulation. Electroencephalogr Clin Neurophysiol 29:225-232. 
Sams M, Alho K, Näätänen R (1983) Sequential effects on the ERP in discriminating two stimuli. Biol Psychol 17:41-58.

Schwartze M, Rothermich K, Schmidt-Kassow M, Kotz SA (2011) Temporal regularity effects on pre-attentive and attentive processing of deviance. Biol Psychol 87:146-151.

Slabu L, Escera C, Grimm S, Costa-Faidella J (2010) Early change detection in humans as revealed by auditory brainstem and middle-latency evoked potentials. Eur J Neurosci 32:859-865.

Takegata R, Morotomi T (1999) Integrated neural representation of sound and temporal features in human auditory sensory memory: an eventrelated potential study. Neurosci Lett 274:207-210.

Ulanovsky N, Las L, Nelken I (2003) Processing of low-probability sounds by cortical neurons. Nat Neurosci 6:391-398.
Ulanovsky N, Las L, Farkas D, Nelken I (2004) Multiple time scales of adaptation in auditory cortex neurons. J Neurosci 24:1044010453.

Umbricht D, Schmid L, Koller R, Vollenweider FX, Hell D, Javitt DC (2000) Ketamine-induced deficits in auditory and visual context-dependent processing in healthy volunteers: implications for models of cognitive deficits in schizophrenia. Arch Gen Psychiatry 57:1139-1147.

Winkler I, Denham SL, Nelken I (2009) Modeling the auditory scene: predictive regularity representations and perceptual objects. Trends Cogn Sci 13:532-540

Zhao L, Liu Y, Shen L, Feng L, Hong B (2011) Stimulus-specific adaptation and its dynamics in the inferior colliculus of rat. Neuroscience 181:163174 . 